Reseña.

Cómo citar:

Editorial: Corporación Universitaria Minuto de Dios - UNIMINUTO.

Recibido: 30 de enero de 2018 Aceptado: 2 de abril de 2018 Publicado: 1 de agosto de 2018

Conflicto de intereses: los autores han declarado que no existen intereses en competencia.
Óscar Murillo Ramírez oscarmur@gmail.com.

Historiador, Universidad Nacional de Colombia. Magíster en Ciencias Políticas, Flacso-Ecuador.

\section{Aprendizajes de construcción de paz en Montes de María}

\author{
Título inglés
}

\section{Título portugués}

Óscar Murillo Ramírez

Pardo, M., Barajas, J., Henao, L. y Huertas, J. (2018). Aprendizajes de construcción de paz en Montes de María. Bogotá: Centro de Investigación y Educación Popular / Programa por la Paz (Cinep/PPP)

A partir de la indagación sobre los mecanismos que permiten transformar las dinámicas violentas que genera el conflicto armado colombiano y, en consecuencia, desatar la construcción de paz territorial, así como delimitar los aprendizajes que las comunidades pueden derivar de sus propias experiencias y relacionamientos con capacidad transformadora, el Equipo Iniciativas de Paz (EIP) del Cinep aborda en este trabajo cuatro iniciativas sociales de paz en la subregión de los Montes de María. Con ello se buscó establecer mediante un marco conceptual y metodológico compartido la manera en que este espacio geográfico, localizado entre los departamentos de Bolívar y Sucre, ha resistido a la histórica presencia de grupos armados ilegales y ha logrado mitigar la reproducción de relacionamientos violentos y los factores estructurales que han contribuido a la persistencia de esta violencia.

El trabajo se encuentra estructurado en tres grandes capítulos, además de una introducción. Es importante señalar que, en esta última, es claro el marco teórico y metodológico al cual recurrió el Equipo de Iniciativas de Paz (EIP) y que se despliega en un primer momento descriptivo para ubicar al lector frente al contexto territorial de la experiencia de paz estudiada. En un segundo momento de corte analítico se conceptualiza el proceso comunitario de transformación de relaciones. Este enfoque se deriva del trabajo de Tarrow, MacAdam y Tilly, quienes otorgan preponderancia a la dinámica entre los actores inmersos en el conflicto y la disputa contingente por el poder a través de sus relaciones. En ese relato descriptivo y analítico se establecen los mecanismos ambientales (referidos al contexto) y específicos (relaciones entre actores y sus 
propias percepciones) en los que se desenvuelven las experiencias de paz de los cuatro casos seleccionados.

En el primer capítulo, el EIP presenta el contexto subregional de los Montes de María a partir de unas breves notas geográficas, la formación histórica territorial y poblacional, destacando la mixtura demográfica actual como un producto de larga duración que incluyó el primer poblamiento y los procesos esclavistas que durante los siglos XVII y XVIII introdujo la Corona española. Estos datos preliminares, antes que un sumario de hechos del pasado, evidencian la manera en que estos territorios y sus gentes han construido una cultura rica en expresiones, la cual se vio cercenada por la irrupción paramilitar que se vivió a finales de la década de los noventa y las transformaciones económicas que mutaron la economía campesina hacia una agroindustrial que se privilegió en el marco del conflicto armado.

En esa reconstrucción introductoria se plantea un aspecto central de la conflictividad social, que constituyó uno de los mecanismos identitarios y de resistencia: el problema de la tierra. El despojo ha sido la principal fuente de acumulación de riquezas en estos territorios del caribe colombiano. A la par, procesos de resistencia y organización surgieron, particularmente, con la constitución y posterior radicalización de la Asociación Nacional de Usuarios Campesinos (ANUC) en la década de los setenta, que movilizó política y socialmente al campesinado por la recuperación de tierras y el impulso del proceso comunitario. Sin embargo, todas estas dinámicas se vieron atravesadas por un relacionamiento violento entre actores, como producto de la radicalización de las élites ante el conflicto sobre sus intereses y la presencia organizada de grupos armados ilegales en distintos momentos y, en muchas ocasiones, de manera simultánea.

Resalta de este capítulo introductorio el doble papel de las mujeres en las dinámicas del conflicto, como víctimas pero también generadoras de resistencia y constructoras de paz territorial a partir del impulso de iniciativas organizadas que buscan establecer marcos de relacionamiento no violento y procesos de reconciliación.

En el capítulo dos, - el más extenso- se presentan los cuatros casos que en los Montes de María constituyen experiencias locales de paz y sus características, con base en el enfoque descriptivo y analítico que se propone establecer los marcos de relacionamiento construidos en el ámbito territorial y sus ejes contextuales (ambientales) y específicos.

Ubicado en el municipio de Morroa (Sucre), el corregimiento de Pichilín es la primera de estas experiencias de paz estudiadas. Tiene como principal característica de abordaje el problema de la tierra, eje de la conflictividad local. Este aspecto, justamente, es una de las variables que explica la presencia histórica de 
organizaciones sociales de orientación campesina que buscaron confrontar la concentración de la tierra y el control político de la zona. La experiencia organizativa más significativa que rememora el EIP es el papel central de la ANUC, particularmente a partir del desmonte de la reforma agraria y la respuesta violenta a las invasiones de tierras que se produjeron desde 1972.

A la par de estas luchas campesinas por la redistribución de la tierra, aunque no derivadas directamente de estas, irrumpieron los primeros grupos armados ilegales. Es importante reafirmar este aspecto, no solo por fidelidad al planteamiento del libro, sino porque, tal como señala el eIP, uno de los desencadenantes del conflicto armado en la subregión de los Montes de María fue precisamente la estigmatización que sobre la población y el territorio se produjo, lo que terminó validando formas de violencia como el paramilitarismo. Sin embargo, el trabajo no desconoce la variada presencia insurgente que incluyó a la Liga Marxista Leninista, el Movimiento de Integración Revolucionaria, y a partir de finales de la década de los ochenta e inicios de los noventa, al Ejército de Liberación Nacional y el Frente 35 de las FARC-EP, hecho que puso en mayor riesgo a la población civil, por cuanto condujo a un estigma de "auxiliadores", pese al explícito rechazo de las comunidades a la presencia de organizaciones armadas ilegales, en particular las FARC-EP.

Tal como se presenta desde la introducción, la existencia de procesos de resistencia a la guerra no obedece a ciertas acciones aisladas, sino al conjunto de relacionamientos en diversos niveles territoriales que establecen las comunidades. En ese sentido, en el caso de Pichilín, se identificó un conjunto de variables que hacen de esta experiencia de paz un ejemplo de la capacidad para mitigar las dinámicas de conflicto violento que pueden tener las comunidades. A la par de esta reconstrucción histórica de las invasiones y la lucha por la tierra, se forjó una identidad campesina como acumulado político que constituyó un mecanismo de solidaridad, el cual se reflejó de manera tangible en el fortalecimiento de procesos sociales como las juntas de acción comunal. Forjar este trabajo colectivo que vinculó a los más jóvenes, y con la intermediación de otros actores, permitió que la formación política se convirtiera en una herramienta para que las comunidades desplegaran acciones colectivas no violentas y lucharan por la reparación colectiva.

Por supuesto estos procesos de resistencia, construcción de paz y transformación de conflictividades, o por lo menos su mitigación, se generan de manera previa a las fuertes rupturas producidas por la violencia, tal como ocurrió con el desplazamiento forzado perpetrado en el corregimiento de Mampuján, municipio de María la Baja (Bolívar). La presencia del Frente 37 de las FARC-EP y el ELN, así como el crecimiento de las extorsiones y secuestros, fueron el catalizador que desató la respuesta violenta de grandes propietarios de tierra y el surgimiento del paramilitarismo, cuya estructura base en el territorio estuvo constituida por el 
Bloque Héroes de Montes de María que perpetró, entre otros hechos victimizantes, el desplazamiento forzado el 10 de marzo de 2000.

Este hecho, aun cuando implicó la destrucción de proyectos de vida, no significó la ruptura total del tejido social. Incluso, es en esta unidad preservada donde el EIP encuentra que se logró gestar una lucha por la reparación colectiva y la restitución de tierras, acompañada, por supuesto, de la construcción de un relacionamiento estratégico que permitió visibilizar esta agenda colectiva y generar acciones de movilización no violenta como las caminatas realizadas hacia Cartagena. Un aspecto relevante de este proceso de resistencia y construcción de paz se halla en la profusa documentación que la comunidad mampujana realizó de su propio caso, lo que le permitió adelantar el proceso de reclamación e interlocución con el Estado.

La "exigibilidad de derechos como experiencia de construcción de paz" (p. 105) que denomina el EIP tuvo en la documentación del caso una de las herramientas más importantes para la reclamación e incidencia. Desde luego, como característica compartida de los casos abordados, el fortalecimiento de procesos sociales y organizativos se convierte en aspecto clave para la transformación de dinámicas de relacionamiento violento hacia otros nuevos, y contribuye a gestar las redes necesarias para generar incidencia, acompañado de estrategias no violentas de acción colectiva como parte de ese escenario de exigibilidad.

Lo que para Pichilín fue la identidad campesina, en el caso de la comunidad de Libertad, localizada en el municipio de San Onofre (Sucre), se expresa a través de la identidad étnica y cultural como eje de la construcción de experiencia de paz. La fuerte presencia de población afrodescendiente tuvo allí un reflejo en la cultura y las dinámicas sociales que fueron, precisamente, las más afectadas con la irrupción y presencia paramilitar entre 1997 y 2004. No obstante, la existencia de ocho cabildos indígenas, así como de los siete consejos comunitarios, evidencia una diversidad étnica que constituyó el eje que permitió la reconstrucción de un nuevo marco de relacionamiento tras la superación relativa de escenarios de violencia.

Un elemento característico del conflicto armado en el territorio que delimita Libertad - y en particular de la presencia paramilitar - fue el sistemático y deliberado ejercicio de regulación de la vida cotidiana y la victimización masiva de mujeres. Sin embargo, es justamente a partir de estas situaciones de violencia que se generan experiencias de paz, particularmente la constituida por organizaciones como Resistencia y Dignificación de las Mujeres de Raza Negra de Libertad, procesos que buscaron, entre otros desatados igualmente por mujeres, reconstruir prácticas ancestrales y culturales que habían sido prohibidas por los paramilitares, por ejemplo los velorios. La urdimbre de esta identidad que recuperó la variable étnica tuvo en expresiones culturales como el bullerengue y el rap uno de sus 
principales componentes y permitió un nuevo marco de relaciones entre los más jóvenes y los adultos de la comunidad. Esto condujo ala defensa de los derechos comunitarios a partir de un variado enfoque que incluyó lo étnico, generacional y de género como lazos constructores de esta iniciativa de paz.

Por último, en este capítulo se aborda la experiencia de paz de Alta Montaña (Carmen de Bolívar), un territorio que por cuenta de las dinámicas geográficas y del conflicto armado terminó dividida por una línea invisible que clasificó a las veredas de acuerdo con el grupo armado ilegal que actuara hegemónicamente. Pese a esto, allí la comunidad logró construir una experiencia de paz, mediante un variado proceso que incluyó el fortalecimiento de liderazgos individuales, prácticas comunitarias como los torneos de fútbol, y el establecimiento de redes de solidaridad más allá de sus propios territorios.

En este caso, el fortalecimiento del proceso organizativo que se inició con la Mesa de Acompañamiento a las Comunidades de Montes de María permitió, además de la visita humanitaria que evidenció los casos de violaciones a los derechos humanos e infracciones al derecho internacional humanitario en el territorio, revitalizar las juntas de acción comunal y con ello, constituir el Movimiento Pacífico de la Alta Montaña como escenario de encuentro de las comunidades antes divididas. El fortalecimiento político, igualmente, se derivó del carácter pacífico de las acciones colectivas desarrolladas, así como los símbolos principales del movimiento, la recuperación de la identidad campesina, territorial y la socialización de experiencias e intercambios comunitarios.

En el tercer capítulo, en un esfuerzo por establecer un marco de entendimiento compartido de las experiencias de paz analizadas, el EIP delimita lo que denomina "lecciones aprendidas regionales" (p. 219). Con ello se busca evidenciar que los pobladores de la región de los Montes de María, además de ser estigmatizados por el conflicto armado que se registró en estos territorios - y que implicó un aislamiento regional y nacional, así como el desconocimiento de las iniciativas de paz que se desarrollaron- también fortalecieron la organización social y comunitaria, recuperaron la identidad étnica y campesina, otorgaron relevancia a la participación de las mujeres alrededor de la construcción de paz.

Asimismo, a manera de recomendación, el libro establece unos principios basados en la experiencia que, de afianzarse, generarían mayores beneficios a las comunidades, tal como ocurre en el caso de la movilización, que ha "[...] demostrado ser un antídoto eficaz contra los señalamientos e indiferencia” (p. 220), la importancia de profundizar la formación política como mecanismo de exigibilidad de derechos y acciones no violentas, y la necesidad de establecer canales de diálogo con la institucionalidad y las élites como herramienta preventiva ante futuros escenarios de relacionamiento violento. 
Sin duda el trabajo realizado por el EIP encuentra en la profusa labor desarrollada mediante talleres y entrevistas una de sus principales fortalezas, que posibilita la ampliación del campo de conocimiento alrededor de la construcción de paz territorial. A partir de allí, es capaz de reconstruir entre fuentes secundarias que se reflejan en la trayectoria de actores armados, contexto histórico y trabajo en terreno los recorridos de los procesos sociales desplegados en los territorios. Las trayectorias comunitarias dejan de ser vistas individualmente al establecer un rígido marco interpretativo y metodológico basado en los elementos de la contienda política. Es esta fidelidad al modelo lo que al final permite sacar unas conclusiones compartidas, no obstante que, como lo señala el mismo texto, paradójicamente las comunidades tienen poco conocimiento de lo que sus pares han desarrollado en materia de paz y fortalecimiento comunitario.

Esta información que aparece tan solo al final permite, justamente, la apertura de un interrogante alrededor de la conciencia adquirida por las comunidades de sus propios procesos y de la subregión. En distintos apartados del texto se afirma, como si fuese un hecho, que las comunidades han interiorizado sus propias experiencias, algo que no queda plenamente demostrado en todos los casos. Es posible creer que en el caso de Alta Montaña los símbolos identitarios remitan a formulaciones no violentas, pero no por ello se están construyendo marcos de cooperación como lo sugieren, precisamente, las diferencias internas que surgieron alrededor de la contienda electoral. Esto habla también de unos lentos procesos de transformación que hacen de la experiencia de paz una práctica política en sí misma que está en proceso de maduración y que, tal como se vislumbra hasta donde establecen los estudios de caso, tienen un largo recorrido aún por realizar. 